\title{
Experience versus expertise - an exploration into the expectations of near-peer teaching
}

\author{
Authors: Laura Stuart and Madhangi Parameswaran
}

\section{Aims}

To ascertain the expectations of medical students on near-peer teaching (NPT) sessions, and the advantages and disadvantages of these compared with their traditional lectures at medical school.

\section{Methods}

Primary data will be collected from a focus group of medical students who have attended one or more NPT sessions run by senior students and foundation doctors as part of the curriculum or organised by the Newcastle Medical Education Society. The students will be recruited using a questionnaire that will provide preliminary data for discussion. General themes of discussion will also be drawn from a questionnaire completed by 35 stage four students about recent near-peer revision lectures. The focus group discussion will be semi-structured, with questions derived from previous data obtained from the questionnaires. A transcript will be written during the discussion. Students will then be asked to complete a short questionnaire at the end of the discussion. The data will then be statistically analysed where appropriate.

\section{Results}

The study is at its recruitment stages. The focus group discussion was to be conducted in February 2018 and its results were not available at the time of writing. From our preliminary questionnaire, we speculate that NPT sessions would be considered more accessible and tutors would seem more approachable in an NPT session. Furthermore, we hypothesise that non-NPT sessions are more suited to laying foundation knowledge and explaining things in detail, whereas NPT tutors can give a broader overview of a topic, which is more suited to revision.

\section{Conclusions}

This study explores the expectations and potential advantages of NPT; its results will hopefully enable NPT, which is increasing in popularity, to be used in teaching opportunities where it will provide the most benefit to medical students.

\section{Conflict of interest statement}

None declared. 